\section{OC37 CHALLENGES AND SUCCESSES OF IMPLEMENTING A PAEDIATRIC EARLY WARNING SYSTEM IN A LIMITED RESOURCE PAEDIATRIC ONCOLOGY SETTING}

${ }^{1}$ Rachel MacDonell ${ }^{*},{ }^{2}$ Trish Scanlan, ${ }^{3}$ Stanley Koe, ${ }^{4}$ kechukwu Okafor, ${ }^{4}$ Claire Ahern, ${ }^{5}$ Adam James. ${ }^{1} R C P I$, Dublin, Ireland; ${ }^{2}$ Muhimbili National Hospital, Dar Es Salaam, Tanzania, United Republic of; ${ }^{3}$ Tallght University Hospital, Dublin, Ireland; ${ }^{4}$ Children's University Hospital, Dublin, Ireland; ${ }^{5}$ Our Lady's Children's Hospital, Dublin, Ireland

\subsection{6/archdischild-2019-epa.36}

Outlining the experience of implementing a paediatric early warning system (PEWS) for Upendo Paediatric Oncology Ward at Muhimbili National Hospital (MNH), Dar Es Salaam, Tanzania.

Background Children on the paediatric oncology ward in Muhimbili National Hospital, Dar es Salaam, were experiencing poor clinical outcomes after common emergencies with $100 \%$ mortality rates for any child requiring CPR. From 2012 to 2015 , a team of Irish paediatric specialists, supported by a Tanzanian NGO called Tumaini la Maisha, provided an annual standard paediatric resuscitation training programme.

Despite these efforts, staff knowledge, skill retention and clinical outcomes did not improve. An observational analysis in 2016 revealed poor vital sign assessment practices, lack of documentation and examples of failure to detect, escalate and respond to child clinical deterioration.

Intervention We designed five bespoke age-specific paediatric observation charts with inbuilt vital sign triggers, escalation pathway, communication guide, 'event record' and a locally appropriate resuscitation course to suit the specific needs of the ward.

Implementation Strategy Over three annual visits from 20162018, PEWS was introduced to Upendo ward with the tailored education programme for medical and nursing staff. Resources including pdf charts, posters, training slide-set and a PEWS implementation policy were provided to facilitate local ownership of the PEWS. Local champions were identified to act as trainers and peer support during the time between visiting faculty visits. In 2018, Paediatric Pocket Guides were introduced as additional aide-mémoires for oncology guidelines and PEWS reference ranges.

Challenges Challenges to implementation of the new charts and escalation behaviours included lack of availability of basic monitoring equipment, low staffing ratios, weekly turnover of medical interns, and insufficient acute care facilities. Ward nursing culture of task-based rather than patient-based care, non-structured handover practices and lack of integrated communication between nurses and the medical team presented the greatest challenge to embedding PEWS.

Successes and Learning By 2018 two new specifically designed courses were developed for MNH. The Paediatric Assessment of Illness, Resuscitation and Stabilisation (PAIRS) and 8-week paediatric oncology nursing course were conducted. PEWS training was central to the ethos of both courses and removed most of the cultural barriers identified above. PEWS and patient-centred care are now standard on the ward with all staff utilising the Paediatric Pocket Guides to supplement their knowledge of PEWS. Early recognition of the acutely unwell and deteriorating child is now a well understood goal of all staff. This initiative shows the importance of audit and local engagement to bring about successful clinical change.

\section{OC38 IMPACT OF MALNUTRITION ON EARLY CHILDHOOD DEVELOPMENT}

Nandita Chattopadhyay* . IQ City Medical College, Durgapur, India

\subsection{6/archdischild-2019-epa.37}

Background Malnutrition and impaired early childhood development continue to exist as two giant childhood health problems, particularly in the developing world. Both conditions often co-exist and exert effect on each other, aggravating the problems. Here we have studied the impact of malnutrition on early childhood development in different age groups, in a marginalized rural community in India, to identify the most vulnerable age group.

Method We screened 837 children aged 0-3 years and 540 aged 3-6 years. Anthropometric measurements (weight, height and mid-arm circumference) were recorded and nutritional status determined by deriving the Z-score for weight for length. Developmental delay was assessed by Denver Development Screening Test II, Trivandrum Development Screening Chart and Amiel-Tyson method of tone assessment.

Result Occurrence of malnutrition was similar in both age groups (33-35\%), with minimum incidence among 0-1 year age group. Incidence of developmental delay was $6.5 \%$ and $3.1 \%$ in $0-3$ year and 3-6 year age groups respectively. In 0 3 years age group Incidence of developmental delay was significantly higher among children with malnutrition ( $p$ value $<0.05)$; no such correlation was seen among the older children.

Conclusion Malnutrition during $1^{\text {st }}$ three years of life, is a major risk factor for poor neuro-developmental outcome. A multi-pronged approach to provide proper nutrition to mother and child, improve health and hygiene and adequate psychomotor stimulation will improve developmental outcome in children.

\section{OC39 THE INCIDENCE OF CONGENITAL HEART DISEASE IN BAKU-AZERBAIJAN. PROSPECTIVE EPIDEMIOLOGY STUDY}

\begin{abstract}
${ }^{1,2,3}$ Andreas Petropoulos* ${ }^{*},{ }^{2}$ Aynur Hudiyeva, ${ }^{2}$ Vali Behbudov, ${ }^{2}$ Gunel Mustafayeva, Nader Guliyev ${ }^{4},{ }^{5}$ Rustlan Huseynov, ${ }^{6}$ Nabil Seyidov. ${ }^{1}$ Great Ormond Street Hospital/CICU, London, UK; ${ }^{2}$ Merkezi Klinika, Baku, Azerbaijan; ${ }^{3}$ The 'Aziz Aliyev' ntional postgraduate and CME medical training center, Baku, Azerbaijan; ${ }^{4}$ Scientific Research Institute of Pediatrics, $\mathrm{MoH}$ of Azerbaijan, Baku, Azerbaijan; ${ }^{5} \mathrm{NICU}$ of Ob/G Institute of $\mathrm{MoH}$ of $\mathrm{Az}, \mathrm{Baku}$, Azerbaijan; ${ }^{6} \mathrm{MoH}$ Azearbaijan, Baku, Azerbaijan
\end{abstract}

\subsection{6/archdischild-2019-epa.38}

Background The incidence of Congenital Heart Disease (CHD) in Azerbaijan has never been calculated with a prospective study, using Echo-2D as a study method

Aim First prospective epidemiology study calculating incidence and types of CHD in Baku, Azerbaijan.

Population-method From June 2016 to August 2018, 2570 term neonates were screened in 2 major maternity units. The screening was randomized to equal females/males with no known previous obstetric alert regarding CHD. Scanning was done by two teams of pediatric cardiologists in echo-2D. Each team was 'blinded' to the findings of each other. All scans were recorded, and a third senior physician reevaluated them. Results From 2570 term neonates of the general population we detected 47 CHD's. From them, 17 were c-CHD and 5, s- 